experts significantly agreed with the elements of debriefing included in OSAD (content validity) and found its instructions clear and intuitive (feasibility). Inter-rater reliability was demonstrated with intra-class correlations of $0.60-0.70$ for 6 of the 8 dimensions of the tool. The internal consistency of OSAD (Cronbach alpha) was 0.79 . OSAD also demonstrated concurrent validity in the form of high correlations with trainees' assessment of debriefings (Pearson $\mathrm{r}=0.71, \mathrm{p}<0.01)$

Conclusion The OSAD tool provides a structured approach to debriefing for paediatricians, which is evidence-based, reliable and valid and relevant to users. OSAD can be used to improve the quality of debriefing after paediatric simulation or after managing a seriously ill child in clinical practise.

\section{G09 DO PARENTS AGREE WITH EXAMINERS WHEN RATING STUDENT COMPETENCE IN EXAMS?}

doi:10.1136/archdischild-2013-304107.022

S Feyereislova, D Nathan. Paediatrics, Nottingham Children's Hospital, Nottingham, UK

\begin{abstract}
Aims To compare examiner and parental ratings of students undertaking a summative clinical assessment of history-taking and examination skills in a ward based setting.
\end{abstract}

Methods Parents of patients participating in the 4th year medical students' ward based assessments were asked for feedback and an evaluation of student performance through validated questionnaire. Students completed a paediatric history and head to toe examination of a child admitted to an acute medical ward within a defined time. The clinical case was presented to examiners (experienced clinicians - paediatric consultants and/or senior Trainees). Examiners ratings were based on student competence in presentation of medical history and examination findings, with observed examination technique and their communication skills during a predetermined clinical role play situation. The latter grading mirrored an $\mathrm{RCPCH}$ scoring system.

Parental ratings were based on the Interpersonal Skills Rating Scale which encompassed their impression of the student's interaction with them and their child. The data was collected in the 2011-2012 academic year. We then correlated aggregated parental scores with examiner rating of the same student.

Results In total, 129 parent evaluations were obtained for 129 students. One hundred and nine parental feedback forms were fully completed and correlated with examiners' scores of matching students. The correlation coefficient for the total scores given by parents and examiners was -0.04 , with intercept at score 22 and the slope of -0.02 . The average score given by parents is 22 (range 16-24). The average score given by examiners is 18 (range 7-24).

Conclusion In general, parental rating does not correlate with clinical examiner rating. Parental ratings of students demonstrate less variation compared to examiner rating scores. Notably, low scoring students were not necessarily considered by parents as underperforming, suggesting students' good communication and interpersonal skills irrespective of technical knowledge.

\section{G10 INSPIRING THE FUTURE OF PAEDIATRICS: A REPORT ON THE PROVISION OF PAEDIATRIC LEARNING EXPERIENCES FOR UK MEDICAL STUDENTS}

doi:10.1136/archdischild-2013-304107.023

S Fosker, R Ives, H Millar, BN Ertansel. Faculty of Medicine, Brighton \& Sussex Medical School, Brighton, UK

Aims To create an event to enhance paediatric knowledge and enthusiasm in the medical student population and establish a forum for students and internationally renowned speakers to share ideas and experiences.

Methods A two day conference was organised by a student paediatric society to welcome over 200 students from all UK medical schools and the local widening access scheme. The conference programme included workshops and lectures by Paediatricians from around the UK. Topics included general paediatric medicine and surgery, in addition to the subspecialties; neonatology, child protection, emergency medicine and child development. Overall, the event provided an opportunity for delegates to experience additional practical and theoretical tuition in paediatrics which is not widely available within most medical schools's curriculum. Additional opportunities were available to present research to visiting lecturers and host academic faculty.

Results 215 delegates attended the conference, $60 \%$ returned feedback forms. Delegates were asked to rank out of five (one being the lowest and five the highest) their experiences of: lectures, workshops, overall conference organisation, relevance and enjoyment. 40 poster titles $(18.6 \%)$ were submitted and presented during the event. Written feedback commended the patient involvement in sessions and the subspecialist topics covered.

\begin{tabular}{ll} 
Abstract G10 Table 1 \\
\hline Feedback categories & Mean Score (1-5) \\
\hline Lectures & 4.45 \\
Workshops & 4.32 \\
Overall relevance & 4.51 \\
Overall enjoyment & 4.63 \\
\hline
\end{tabular}

Conclusion The content of the lectures and workshops were well received and the overall enjoyment of the event was ranked highly. Sessions in subspecialist topics and those which included patients were particularly acclaimed. This could be secondary to limited exposure to such opportunities at medical school, which suggests that in the future the event programme should further focus on incorporating these sessions. Additionally, it was identified that students endeavour to be involved in academic paediatric research early in their educational career. Analysing the feedback provided evidence that students desire more exposure to paediatrics within their medical training. This emphasises the need for such an event to continue on an annual basis in order to further raise the profile of paediatrics and provide learning opportunities in addition to medical school curriculum.

\section{G11 PROCEDURAL OPPORTUNITIES FOR PAEDIATRIC TRAINEES}

doi:10.1136/archdischild-2013-304107.024

'K Wallace, ${ }^{2} \mathrm{D}$ Cochran. 'Neonatal Department, Wishaw General Hospital, Wishaw, UK, ${ }^{2}$ Neonatal Department, Southern General Hospital, Glasgow, UK

Aim Trainees in paediatrics are required to become competent at a number of practical procedures but often express concern at lack of opportunities. We aimed to survey the number of practical procedures undertaken in 3 months, in two level 3 neonatal units in Scotland, and how these opportunities are distributed amongst trainees.

Method Opportunities occurring for 7 procedures were documented during a 3 month period in 2 separate units, an antenatal referral centre for cardiac and surgical anomalies and a large district general hospital. Procedures recorded were intubation, umbilical arterial and venous lines (UAC, UVC), peripheral arterial lines, long lines, chest drains and lumbar punctures. Data was collected on the number of opportunities, training status of practitioner and clinical characteristics of the baby. 